\title{
Transatlantica
}

Revue d'études américaines. American Studies Journal

1 | 2017

Morphing Bodies: Strategies of Embodiment in Contemporary US Cultural Practices

\section{La guerre de Sécession enrôlée dans le débat politique français}

\section{Nicolas Martin-Breteau}

\section{OpenEdition}

\section{Journals}

Édition électronique

URL : https://journals.openedition.org/transatlantica/9013

DOI : 10.4000/transatlantica.9013

ISSN : 1765-2766

Éditeur

Association française d'Etudes Américaines (AFEA)

Référence électronique

Nicolas Martin-Breteau, «La guerre de Sécession enrôlée dans le débat politique français »,

Transatlantica [En ligne], 1 | 2017, mis en ligne le 29 novembre 2018, consulté le 20 mai 2021. URL :

http://journals.openedition.org/transatlantica/9013 ; DOI : https://doi.org/10.4000/transatlantica. 9013

Ce document a été généré automatiquement le 20 mai 2021.

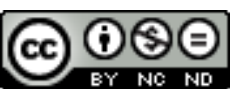

Transatlantica - Revue d'études américaines est mise à disposition selon les termes de la licence Creative Commons Attribution - Pas d'Utilisation Commerciale - Pas de Modification 4.0 International. 


\title{
La guerre de Sécession enrôlée dans le débat politique français ${ }^{1}$
}

\author{
Nicolas Martin-Breteau
}

\section{RÉFÉRENCE}

Le Figaro-Histoire, « La guerre de Sécession : la véritable histoire », n 35, décembre 2017janvier 2018

1 La falsification de l'histoire à des fins partisanes n'est pas nouvelle (Citron ; Offenstadt et Meyran ; CVUH ; Aggiornamento hist-géo). Dernier exemple en date, la publication par le magazine Le Figaro-Histoire d'un dossier d'une soixantaine de pages consacré à la « véritable histoire » - entendre non « manichéenne » (46) - de la guerre de Sécession (1861-1865). Le dossier avance une interprétation dépassée de la guerre de Sécession qui n'a plus cours depuis longtemps aux États-Unis, si ce n'est dans les milieux d'extrême droite. Le dossier reprend en effet les principaux éléments de réhabilitation du Sud esclavagiste forgés dès le lendemain de la défaite en 1865. Si ce dossier d'un hebdomadaire français doit attirer notre attention, c'est que l'instrumentalisation d'une histoire étrangère y joue au service d'un dessein politique national. Le dossier montre en effet une internationalisation de stratégies mémorielles réactionnaires visant à protéger une identité « occidentale » considérée comme menacée.

\section{La relativisation de l'esclavage}

2 Le dossier jette d'abord le doute sur les causes de la guerre de Sécession. L'esclavage n'aurait au maximum été, d'après l'avocat et essayiste Jean-Louis Thiériot, rédacteur de l'article d'ouverture, que le «détonateur» de la guerre, mais pas son «enjeu»: l'esclavage n'a «pas été la seule cause, tout au plus l'une de ses causes » $(42,46-47)$. Autrement dit, l'esclavage aurait été une cause de la guerre bien moins importante que 
les «profondes différences économiques, sociales et culturelles » entre Nord et Sud comme si toutes ces différences n'étaient pas directement liées à l'esclavage (47).

En réalité, comme le montre une production scientifique abondante, l'esclavage fut la cause fondamentale de la guerre de Sécession précisément en raison de son immense influence économique, politique, sociale et culturelle dans les États-Unis du premier XIX ${ }^{\mathrm{e}}$ siècle (Baptist; Beckert). Les États sécessionnistes n'en faisaient d'ailleurs pas mystère. Leurs Déclarations de sécession justifièrent leur rébellion en usant de la question de l'esclavage, comme le Mississippi en janvier 1861 qui déclarait d'emblée : « Notre position est entièrement identifiée à l'institution de l'esclavage » car « un coup porté à l'esclavage est un coup porté au commerce et à la civilisation » (State of Mississippi). La même année, le vice-président de la Confédération sudiste, Alexander H. Stephens, affirmait que la « pierre angulaire » de la nouvelle nation sudiste était « la grande vérité selon laquelle le Noir n'est pas l'égal de l'homme blanc, que l'esclavage, la subordination à la race supérieure, est sa condition naturelle et normale " (Stephens). Bien plus, les historiens ont clairement montré que le but du Sud, pendant et avant la guerre, fut de défendre et, si possible, d'étendre l'esclavage à tous les États-Unis, à toute l'Amérique, voire au monde entier (Johnson 280-420; Karp).

Pourtant, l'esclavage n'aurait que «fissuré » ce que Jean-Louis Thiériot présente comme «l'exemplarité, la singularité, l'exceptionnalité » de la «jeune nation» (47). C'est peu dire que les quatre millions d'esclaves et le demi-million de Noirs libres aux États-Unis en 1861 n'ont pas interprété cette histoire de la même façon. Grâce à leur fuite massive des plantations et à leur participation active au conflit, les AfricainsAméricains jouèrent un rôle décisif dans la victoire du Nord et l'abolition de l'esclavage. À la fin de la guerre, près de 200000 Africains-Américains servaient dans l'armée du Nord et 40000 y perdirent la vie. En ce sens, la guerre de Sécession fut une gigantesque révolution des esclaves, la plus grande de l'histoire de l'humanité (Berlin et al., 1982; 1992 ; 1998 ; Hahn, 2009 55-114). Contrairement aux auteurs du dossier, les AfricainsAméricains ne se sont jamais mépris sur les causes de la guerre (Quarles; Berlin; Sinha).

Dès la défaite sudiste, la relativisation de l'esclavage dans le déclenchement du conflit représenta un aspect de la mythologie de la « cause perdue " qui cherchait à réhabiliter le Sud esclavagiste en glorifiant la noblesse - dans tous les sens du terme - de son combat. Publié en 1866, l'ouvrage d'Edward A. Pollard, The Lost Cause: A New Southern History of the War of the Confederates, a défini le cadre de ce discours violemment réactionnaire et raciste. Dès le titre, l'ouvrage se présente comme une histoire "approuvée par les chefs confédérés les plus distingués». Pollard affirme ainsi que la guerre mit face à face "deux nations aux civilisations opposées» de sorte que l'esclavage n'y aurait constitué qu'un "simple incident", d'après lui faussement considéré comme le principal sujet de «controverse» (47-48). Pour les tenants de la cause perdue, considérer l'esclavage comme la cause principale de la guerre participait d'une vaste entreprise de diffamation coordonnée par le Nord, jaloux de la grandeur et du raffinement de la civilisation du Sud (Blight ; Foster ; Vann Woodward ; Foner, 1988 ; Barreyre et Schor 1-17, 138-151). 


\section{La glorification des héros sudistes}

6 Ce premier point permet ensuite aux auteurs du dossier de décrire une guerre ne mettant aux prises que des hommes blancs, tous valeureux et chevaleresques. Dans les deux camps, des "héros » mériteraient l'admiration. Historiquement, la glorification de la supériorité morale des chefs de guerre esclavagistes et de leurs soldats fut un ressort essentiel de la mythologie de la cause perdue permettant de masquer la réalité des objectifs de la guerre menée par la Confédération sudiste (Blight). Ce sont des héros, disait déjà Pollard en 1866, qui, "au milieu des calamités publiques et de leur maigre fortune personnelle, laissent derrière eux le souvenir de nobles actions et un héritage de gloire immortel » (751).

7 Dans le dossier du Figaro-Histoire, Jefferson Davis, président de la Confédération esclavagiste, devient sous la plume de Vincent Bernard un "riche planteur", « intelligent » et "soucieux à l'excès de sa propre dignité », "un homme de grande expérience et un parfait représentant des "valeurs sudistes" » (62). Nathan Bedford Forrest, esclavagiste, criminel de guerre et fondateur du Ku Klux Klan qui détruisit par le terrorisme la démocratie interraciale de l'après-guerre, est présenté par le même auteur comme un « héros populaire » loué pour sa " personnalité forte » et son " génie militaire" (64). Dans le premier article du dossier, Jean-Louis Thiériot ramène d'ailleurs les exactions racistes du KKK à des «aventures de desperados » menées par des résistants « déterminés » (53).

Robert E. Lee, le commandant en chef des armées esclavagistes, occupe une place toute particulière dans cette hagiographie des leaders sudistes. Sa légende dorée insiste à l'envi sur sa distinction raffinée et son génie miliaire. Dans le dossier du Figaro-Histoire, Lee est ainsi présenté par Vincent Bernard comme un "parfait gentleman", " impressionnant de stature et de distinction", "aimable et charmeur ", bref " une idole quasi incontestée » $(54,57)$. De même, dans la bibliographie qui conclut le dossier, le résumé du livre de Vincent Bernard consacré à Robert $\mathrm{E}$. Lee le présente comme un " homme de convictions ", "brillant capitaine ", «bel homme, père de famille, fervent chrétien » (98; Bernard). En conséquence, l'auteur de l'article affirme sans prendre visà-vis de son objet la distance critique nécessaire que la « figure respectée » de Lee serait aujourd'hui injustement "submergée par la mémoire, il est vrai longtemps occultée, associée aux Africains-Américains et à l'esclavage, qui refuse de le considérer au-delà de son rôle de champion d'un monde esclavagiste honni » $(57$; voir par contraste Foner, 2017).

$9 \mathrm{Au}$ lendemain de la guerre, des centaines de monuments, de statues et d'écoles furent érigées dans le Sud à la gloire de ces personnalités sudistes - au premier rang desquelles Robert E. Lee. L'édification de ces statues s'inscrivait dans un projet politique réactionnaire destiné à marquer la permanence de la suprématie blanche dans l'espace public même après l'abolition de l'esclavage. Sous l'influence d'organisations paramilitaires puissantes comme le Ku Klux Klan, ces monuments furent principalement installés dans les années 1900-1920 et 1950-1960, deux périodes de violente contre-révolution raciale aux États-Unis, au cours desquelles les nostalgiques du Sud d'avant-guerre cherchèrent à renforcer la ségrégation raciale comme le seul régime social pouvant succéder à l'esclavage. Le déboulonnage actuel des statues de personnalités confédérées, amèrement déploré par Jean-Louis Thiériot (46), 
est incompréhensible sans le rappel de ce contexte historique (SPLC; Cobb; Schuessler ; Cox).

\section{Une célébration nostalgique du Sud esclavagiste}

10 Sur ces bases, le dossier s'engage enfin dans une célébration nostalgique de la société sudiste d'avant-guerre, en paraphrasant, plus qu'en les analysant, les deux productions culturelles emblématiques de la cause perdue : le roman Autant en emporte le vent de Margaret Mitchell paru en 1936 et le film éponyme de Victor Fleming sorti en 1939 (Dickey). Le projet politique de la cause perdue s'appuyait en effet sur une présentation idéalisée et romantique de la vie agraire dans le Sud au temps de l'esclavage. L'insistance sur le raffinement culturel $\mathrm{du}$ Sud fut une constante du discours esclavagiste destiné à dissimuler la brutalité d'une société reposant tout entière sur la mise en esclavage de millions d'hommes, de femmes et d'enfants. Née à Atlanta en 1900, Margaret Mitchell fut, comme ses contemporains blancs, nourrie de cette idéologie fondatrice du suprémacisme blanc.

11 Ainsi, sur fond de demeures aristocratiques bordées de champs de coton, de magnolias en fleurs et de robes à crinoline, le film Autant en emporte le vent s'ouvre par un texte convoquant le mythe d'une terre de chevaliers galants et de gentes dames qu'on ne pourra plus désormais approcher que dans les livres car, comme le «souvenir d'un rêve ", ce « beau monde » n'est plus qu'une « civilisation emportée par le vent » - ceci avant d'enchaîner par une suite quasiment ininterrompue de plans véhiculant des stéréotypes grossiers sur l'infériorité comique des Noirs et la malfaisance catastrophique des Nordistes.

Jean-Louis Thiériot reprend à son compte ce type de discours en exaltant la vision agrarienne du Sud esclavagiste présenté comme «le pays magique de la nostalgie du monde d'avant, d'avant la chute, d'avant l'industrie» (53). En contrepoint, l'article reproduit une caricature hostile au sénateur républicain, promoteur des droits des Noirs et ancien officier de l'armée du Nord, Carl Schurz (1829-1906), laconiquement présenté comme l'un de ces innombrables "aventuriers » et "profiteurs cupides et sans scrupules » venus persécuter les Sudistes « accablés par la défaite » (52-53).

Dans la contribution de Geoffroy Caillet, cette élégie paternaliste à la gloire du bon vieux temps de l'esclavage qu'est Autant en emporte le vent est présentée comme un film "sensible " au sort des esclaves au prétexte que les maîtres leur témoigneraient une "sollicitude parfaite" (84) - argument employé ad nauseam au XIX ${ }^{\mathrm{e}}$ siècle pour légitimer les bienfaits de l'esclavage pour les Noirs. Bien qu'il qualifie de " partiale » la représentation de l'esclavage dans le film, l'auteur de l'article, et rédacteur en chef du Figaro-Histoire, salue néanmoins le dévouement des esclaves à leurs " maîtres modèles ", selon la vision du monde sudiste où les Noirs doivent occuper avec joie une place inférieure et soumise dans un ordre racial à la fois naturel et éternel (88). L'article va jusqu'à affirmer que cette vision idéalisée de l'ordre racial de l'esclavage aurait « réussi l'exploit de donner une aura universelle au Sud vaincu » ( 88 ; je souligne). Là encore, sans contextualisation rigoureuse, l'auteur en arrive finalement à déclarer qu'Autant en emporte le vent serait injustement devenu "l'otage du communautarisme moderne ", menacé de " purgatoire " par une " mémoire exacerbée » (88). 
une stricte équivalence entre la prétendue «brutalité » des "profiteurs du Nord, affairistes et aventuriers " et la violence raciste inouie du film Naissance d'une nation (D.W. Griffith, 1915) qui célèbre l'action rédemptrice des chevaliers du Ku Klux Klan assujettissant les Noirs à l'ordre racial ancien par le terrorisme armé. En ce sens, Naissance d'une nation ne serait qu'une réponse logique aux « "radicaux" du Congrès » et montrerait " combien les blessures restent à vif cinquante ans après la fin du conflit " (53 ; voir aussi 87-88). Dans le même ordre d'argument, Jean-Louis Thiériot expose, sans les contextualiser ni les critiquer, les arguments classiques des partisans de l'esclavage depuis le XIX ${ }^{e}$ siècle selon lesquels les « ouvriers libres de Nouvelle-Angleterre sont davantage asservis que les Noirs" - ce qui ferait ainsi de l'esclavage un "système humanitaire » (49).

\section{La guerre de Sécession, une histoire française?}

En réactivant ces trois piliers de la cause perdue - relativisation de l'esclavage glorification des héros sudistes et célébration de la culture sudiste -, le dossier du Figaro-Histoire obéit in fine à un dessein politique. Une historiographie rétrograde de la guerre de Sécession y est enrôlée au service de prises de position politiques dans le débat français sur la définition de ce que d'aucuns appellent le «roman national ». En ce sens, l'histoire et la mémoire de la guerre de Sécession ne concernent pas que les États-Unis, mais également la France d'aujourd'hui. Ironiquement, une histoire étrangère sert ici à défendre l' « identité nationale » française.

Dans l'article d'ouverture, Jean-Louis Thiériot, alors premier vice-président Les Républicains (LR) du Conseil départemental de Seine-et-Marne et aujourd'hui député LR de Seine-et-Marne, explique en quoi le dossier vise implicitement la «flambée » de «fureur mémorielle » qui traverserait aujourd'hui les États-Unis sur la question de l'esclavage, et qui contaminerait la France (46).

17 Au moment de sa parution, le dossier s'inscrivait donc à la croisée de deux débats simultanés: le débat aux États-Unis sur le déboulonnage de monuments publics célébrant les anciens défenseurs de l'esclavage ; et le débat lancé en septembre 2016 par le Conseil représentatif des associations noires de France (CRAN) sur les symboles esclavagistes dans l'espace public français. Le CRAN visait notamment le nom de collèges et de lycées nommés en l'honneur de Jean-Baptiste Colbert, qui participa à l'institutionnalisation de l'esclavage dans les colonies françaises notamment au moyen du «Code noir» (1685). Le CRAN proposait de remplacer le nom de Colbert par celui "de ces héros, noirs ou blancs, bien souvent méconnus, qui luttèrent contre l'esclavage » (Tin).

Proposer une stricte équivalence entre Nord et Sud pendant la guerre de Sécession permet au dossier du Figaro-Histoire de réfuter d'emblée toute légitimité à ce type de demande. Sans mise en perspective historiographique, ce relativisme est validé dans le dossier sous la forme d'un simple mouvement de balancier, idéologiquement neutre: l'historien André Kaspi, maire-adjoint LR de la ville de Saint-Maur-des-Fossés (Val-deMarne), affirme ainsi que, dans les années 1870, "c'est la réconciliation qui l'emporte »; mais dans les années 1960, « le Sud redevient coupable » (75).

19 Ce type de propos n'explique pas comment, une génération seulement après 1865 , la continuation de la guerre de Sécession sur le front mémoriel fut remportée par les suprémacistes blancs qui parvinrent à imposer à tout le pays leur vision des causes, du 
déroulement et des conséquences de la guerre. Jusqu'au mouvement pour les droits civiques des années 1950 et 1960 au moins, l'historiographie dominante (blanche) de la guerre de Sécession ne fit que proposer des variations sur le thème raciste de la cause perdue dont la version la plus répandue fut popularisée par la Dunning School, du nom du professeur de l'université Columbia, William Archibald Dunning (1857-1922) (Dunning; pour une réfutation pionnière, voir Du Bois).

Pourtant, dans les années 1860, la plupart des Français ne se trompaient pas sur l'histoire dont ils étaient les témoins, considérant que la guerre de Sécession avait été livrée au sujet de l'esclavage. En France, l'immense popularité d'Abraham Lincoln au moment de son assassinat en avril 1865 explique le succès de la souscription publique lancée en son honneur. À l'époque, Lincoln n'était pas d'abord considéré comme un chef de guerre ou comme le sauveur de l'Union, mais comme un président politiquement et moralement courageux qui, devant la gravité des événements et la pression des abolitionnistes, finit par assurer la libération des esclaves (Foner 2010). En ce sens, quinze ans après l'abolition de l'esclavage en France, nombreux furent les Français qui, comme Victor Hugo, virent en lui un martyr mort pour la cause de la liberté.

21 Ce qu'il faut donc analyser et comprendre, c'est l'immense travail contrerévolutionnaire opéré par les tenants du Vieux Sud esclavagiste pour imposer leur interprétation de l'histoire de la guerre au lendemain de la défaite militaire. Par exemple, le fait que les Américains nomment encore aujourd'hui cette guerre la "guerre civile ", plutôt que la "guerre de Sécession » ou la "guerre de la Rébellion " comme le faisaient les Nordistes, montre à quel point le Sud a réussi, au cours des décennies suivant la fin des hostilités, à imposer sa vision d'un conflit célébrant deux ennemis qui, finalement, auraient chacun eu raison (Blight 5 ; Hahn, 2016 4).

Ce qu'il faut également analyser et comprendre, c'est la façon dont ces représentations mémorielles politiquement partisanes et scientifiquement erronées se diffusent à nouveau. Cette lecture de la guerre de Sécession est aujourd'hui vivace dans les milieux d'extrême droite américains, mais aussi français. Ainsi, l'article d'ouverture du dossier, dû à Jean-Louis Thiériot, est clairement inspiré des écrits de l'essayiste d'extrême droite Alain Sanders $(2012$; $2015 ;$ 2017c). L'un des derniers ouvrages de Sanders, consacré à John Wilkes Booth présenté comme l'assassin du "tyran» Lincoln par amour du Sud (2017b), est d'ailleurs cité en bibliographie du dossier (100).

Emblématiques de cette mouvance idéologique, les dizaines d'ouvrages d'Alain Sanders s'emploient à dénoncer la prétendue "désinformation " existant autour de sujets comme la guerre de Sécession, Marie-Antoinette, l'OAS ou Jean-Marie Le Pen. Ces ouvrages sont tous publiés par des maisons d'éditions marquées à l'extrême droite, comme Fol'fer, Godefroy de Bouillon, Pardès ou Clovis. Dans le quotidien d'extrême droite Présent, après un jeu de mots révélateur des liens qui selon lui unissent les situations française et états-unienne - «Je suis (Char) Lee!»-, Alain Sanders a récemment pris la défense du meurtrier de la jeune Heather Heyer, tuée le 12 août 2017 lors des manifestations organisées par divers mouvements suprémacistes blancs s'opposant au démontage de la statue de Robert E. Lee à Charlottesville en Virginie (2017a). 


\section{Un « combat culturel » international ?}

Afin de contrer ce qu'il nomme la "repentance occidentale", le dossier du FigaroHistoire manipule donc une histoire étrangère à des fins politiques nationales. Pour intervenir dans les débats mémoriels français sur la colonisation et l'esclavage, il est hautement significatif que le dossier utilise les ressorts de la cause perdue. Cette idéologie fut l'aspect culturel d'un projet politique global cherchant à rétablir, par tous les moyens, la domination raciale blanche sur les anciens esclaves. Comme l'affirmait Edward Pollard, la cause perdue devait permettre au Sud vaincu de mener une "guerre des idées » au moyen de "l'arme des arguments» pour remporter la victoire sur le terrain de la mémoire (Pollard 750).

Ainsi, ce dossier du Figaro-Histoire ne représente-t-il qu'un des nombreux fronts de ce que le directeur adjoint du Figaro Magazine et présentateur d'émissions sur la chaîne L'Histoire Jean-Christophe Buisson désigne explicitement comme un «combat culturel » destiné à contrer les « mensonges » de l'histoire universitaire. Par exemple, le 28 décembre 2017, à propos de la sortie du Figaro Magazine des 29-30 décembre 2017, dont le dossier central dirigé par Jean Sévillia est intitulé «Histoire de France: Arrêtons les mensonges » (notamment sur Colbert), Jean-Christophe Buisson publiait ce tweet: "Contrairement à d'autres hebdos, @Le_Figaro Magazine ne prend pas ses quartiers d'hiver car le combat culturel n'attend pas. En kiosques, dès ce vendredi ». Aussi est-ce à juste titre que ce type de discours sur l'histoire a déjà été analysé comme une " offensive culturelle», voire une " guerre culturelle » ignorant volontairement le travail de décennies de recherche scientifique (offenstadt et Meyran 25).

Cependant, de façon nouvelle, ce dossier du Figaro-Histoire tend à montrer que ce « combat culturel» ne se structure pas seulement à l'échelle française, mais aussi à l'échelle internationale. Plus que d'autres thèmes, l'histoire de l'esclavage permet une telle internationalisation des polémiques mémorielles. L'esclavage fut en effet une institution globale dans le monde atlantique entre la fin du XV siècle et la fin du XIX siècle. La France, qui déporta 1,4 million d'Africains vers ses colonies d'Amérique, a très largement participé à cette histoire, tout en en tirant des bénéfices économiques et politiques colossaux. L'historiographie a fait sur ces questions de remarquables progrès ces dernières années (Ismard).

Ce dossier du Figaro-Histoire appelle donc à approfondir les recherches actuellement en cours sur l'esclavage et la colonisation, mais aussi à renforcer l'enseignement de l'histoire de ces questions notamment au collège et au lycée. D'une part, il est évident qu'un tel dossier n'est possible que parce que l'histoire de l'esclavage, à la différence de l'histoire d'autres crimes contre l'humanité, reste relativement méconnue dans l'opinion publique française (Boilley). Qui irait proposer une histoire non « manichéenne » de la Seconde Guerre mondiale en relativisant les causes raciales de la Shoah ou en célébrant les manières distinguées des chefs de guerre nazis?

D'autre part, l'instrumentalisation transnationale des mémoires, notamment sur les questions raciales, doit stimuler l'étude de ces phénomènes par les sciences sociales (Weil et Dufoix; Vidal; Larcher; Aje et Gachon). Que Le Figaro-Histoire vise la " repentance occidentale » - et non pas simplement la repentance française - est un indice patent de l'internationalisation grandissante des questions identitaires dans les sociétés multiculturelles contemporaines. Par exemple, les origines françaises de certains éléments de l'idéologie néo-raciste de l'extrême droite américaine et 
européenne - le "grand remplacement » à l'origine d'un "génocide anti-blanc » - ont récemment pu être mises en évidence (Williams ; Williams et Behrer). Simultanément, des représentants de l'extrême droite aux États-Unis, au premier rang desquels l'ancien conseiller de Donald Trump Stephen Bannon, travaillent activement à des rapprochements avec les partis européens de cet univers idéologique. Le Rassemblement national (ex-Front National) de Marine Le Pen devrait ainsi prochainement adhérer à la fondation de Stephen Bannon, The Movement, qui cherche à fédérer les extrêmes droites européennes et états-uniennes (Nougayrède ; Aliot et Sengès).

À l'heure où l'on parle d'une Fondation pour la mémoire de l'esclavage en France, des dossiers tels que celui du Figaro-Histoire n'invitent pas à la pensée critique sur ces questions (Zinsou et Bayle; Ayrault). Aussi, parce qu'il entend influencer de façon scientifiquement erronée le débat public sur des sujets hautement polémiques, le dossier du Figaro-Histoire doit alerter la vigilance des historiennes et des historiens, et les inciter à intervenir dans l'espace public. Plus que jamais, il leur revient de participer à la définition d'un cadre d'analyse rigoureux pour les questions de notre temps.

\section{BIBLIOGRAPHIE}

AJE, Lawrence, et Nicolas GACHON, éd. La mémoire de l'esclavage. Traces mémorielles de l'esclavage et des traites dans l'espace atlantique. Paris : L'Harmattan, coll. « Chemins de la Mémoire », 2018.

ALIOT, Louis, et Gilles SENGÈS. « Steve Bannon a fait gagner Trump et si demain il peut nous aider à faire de même... ». L'Opinion, 17 septembre 2018, lopinion.fr/edition/international/louisaliot-rn-steve-bannon-a-fait-gagner-trump-si-demain-il-peut-nous-161860. Page consultée le 22 septembre 2016

AGGIORNAMENTO HIST-GEO. aggiornamento.hypotheses.org/. Voir notamment « Introduction du dossier Figaro-Histoire : "La vérité sur l'Histoire à l'école" ou les mensonges du Figaro ", 15 octobre 2012, aggiornamento.hypotheses.org/1039. Page consultée le 22 septembre 2016.

AYRAULT, Jean-Marc. « Pour la création d'une Fondation pour la mémoire de l'esclavage ». Libération, 20 novembre 2017, http://www.liberation.fr/debats/2017/11/20/pour-la-creation-dune-fondation-pour-la-memoire-de-l-esclavage_1611347. Page consultée le 22 septembre 2016.

BAPTIST, Edward E. The Half Has Never Been Told: Slavery and the Making of American Capitalism. New York : Basic Books, 2014.

BARREYRE, Nicolas, et Paul SCHOR. De l'émancipation à la ségrégation, le Sud des États-Unis après la guerre de Sécession. Paris : PUF/CNED, 2009.

BECKERT, Sven. Empire of Cotton: A Global History. New York : Alfred A. Knopf, 2014.

BERLIN, Ira. The Long Emancipation: The Demise of Slavery in the United States. Cambridge, MA. : Harvard University Press, 2015.

BERLIN, Ira, Barbara J. FIELDS, Steven F. MILLER, Joseph P. REIDY et Leslie S. ROWLAND. Slaves No More: Three Essays on Emancipation and the Civil War. New York : Cambridge University Press, 1992. 
BERLIN, Ira, Thavolia GLYMPH, Steven F. MILLER, Joseph P. REIDY, Leslie S. ROWLAND et Julie SAVILLE, éd. Freedom: A Documentary History of Emancipation, 1861-1865. Series II: The Black Military Experience. New York : Cambridge University Press, 1982.

BERLIN, Ira, Joseph P. REIDY et Leslie S. ROWLAND, éd. Freedom's Soldiers: The Black Military Experience in the Civil War. New York : Cambridge University Press, 1998.

BERNARD, Vincent. Robert E. Lee. La légende sudiste. Paris : Perrin, 2014.

BLIGHT, David W. Race and Reunion: The Civil War in American Memory. Cambridge, MA. : Harvard University Press, 2001. Un article récent en résume les grandes lignes : David W. BLIGHT. «The Civil War Isn't Over ». The Atlantic, 8 avril 2015, theatlantic.com/politics/archive/2015/04/thecivil-war-isnt-over/389847/. Page consultée le 22 septembre 2016.

BOILLEY, Pierre. « Un enseignement ouvert au monde? Carences françaises et frustrations de mémoires ». L'Afrique de Sarkozy. Un déni d'histoire. Éd. Jean-Pierre Chrétien. Paris : Karthala, 2008, p. 133-154.

CITRON, Suzanne. Le Mythe national. L'histoire de France revisitée. Ivry-sur-Seine : L'Atelier, 2017 [1987].

COBB, Jelani. « The Battle over Confederate Monuments in New Orleans ». The New Yorker, 12 mai 2017, newyorker.com/news/daily-comment/the-battle-over-confederate-monuments-in-neworleans. Page consultée le 22 septembre 2016.

COX, Karen L. « The whole point of Confederate monuments is to celebrate white supremacy ». The Washington Post, 16 août 2017, washingtonpost.com/news/posteverything/wp/2017/08/16/ the-whole-point-of-confederate-monuments-is-to-celebrate-white-supremacy/. Page consultée le 22 septembre 2016 .

CVUH (Comité de vigilance face aux usages publics de l'histoire). cvuh.blogspot.fr/.

DICKEY, Jennifer W. A Tough Little Patch of History: Gone with the Wind and the Politics of Memory. Fayetteville : The University of Arkansas Press, 2014.

DU BOIS, W. E. B. Black Reconstruction in America, 1860-1880. New York : The Free Press, 1998 [1935].

DUNNING, William Archibald. Reconstruction, Political and Economic, 1865-1877. New York et Londres : Harper and Brothers, 1907.

FONER, Éric. Reconstruction: America's Unfinished Revolution, 1863-1877. New York : Harper and Row, 1988.

---. The Fiery Trial: Abraham Lincoln and American Slavery. New York : W. W. Norton, 2010.

---. « The Making and the Breaking of the Legend of Robert E. Lee ». New York Times, 28 août 2017, nytimes.com/2017/08/28/books/review/eric-foner-robert-e-lee.html. Page consultée le 22 septembre 2016.

FOSTER, Gaines M. Ghosts of the Confederacy: Defeat, the Lost Cause, and the Emergence of the New South, 1865-1913. New York : Oxford University Press, 1987.

HAHN, Steven. The Political Worlds of Slavery and Freedom. Cambridge, MA. : Harvard University Press, 2009.

---. A Nation Without Borders: The United States and Its World in an Age of Civil Wars. New York : Viking, 2016.

ISMARD, Paulin. « Écrire l'histoire de l'esclavage. Entre approche globale et perspective comparatiste ». Annales. Histoire, Sciences Sociales, vol. 72, n 1, 2017, p. 7-43. 
JOHNSON, Walter. River of Dark Dreams: Slavery and Empire in the Cotton Kingdom. Cambridge, MA. : Harvard University Press, 2013.

KARP, Matthew. This Vast Southern Empire: Slaveholders at the Helm of American Foreign Policy. Cambridge, MA. : Harvard University Press, 2017.

LARCHER Silyane. L'Autre citoyen. L'idéal républicain et les Antilles après l'esclavage. Paris : Armand Colin, 2014.

NOUGAYRÈDE, Natalie. « Steve Bannon is on a far-right mission to radicalise Europe ». The Guardian, 6 juin 2018, theguardian.com/commentisfree/2018/jun/06/steve-bannon-far-rightradicalise-europe-trump?CMP=share_btn_tw. Page consultée le 22 septembre 2016.

OFFENSTADT, Nicolas, et Régis MEYRAN. L'histoire, un combat au présent. Paris : Éditions Textuel, 2014.

POLLARD, Edward A. The Lost Cause: A New Southern History of the War of the Confederates: Comprising a Full and Authentic Account of the Rise and Progress of the Late Southern Confederacy-the Campaigns, Battles, Incidents, and Adventures of the Most Gigantic Struggle of the World's History: Drawn from Official Sources, and Approved by the Most Distinguished Confederate Leaders. New York : E. B. Trat \& Co., 1866.

QUARLES, Benjamin. The Negro in the Civil War. Boston : Beacon Press, 1953.

SANDERS, Alain. La désinformation autour de la guerre de Sécession. La Chaussée d'Ivry : Atelier Fol'fer, 2012.

---. Robert E. Lee, Paris : Éditions Pardès, coll. « Qui suis-je ?», 2015.

---. « Autour des événements de Charlottesville - Le coup de gueule d'Alain Sanders », Présent, 23 août 2017, https://present.fr/2017/08/23/autour-evenements-de-charlottesville-coup-degueule-dalain-sanders/. Page consultée le 22 septembre 2018.

---. Il a tué Lincoln! J. W. Booth, le Brutus des Sudistes. La Chaussée d'Ivry : Atelier Fol'fer, 2017.

---. La désinformation autour d'Abraham Lincoln. La Chaussée d'Ivry : Atelier Fol'fer, 2017.

SCHUESSLER, Jennifer. « Historians' Question Trump's Comments on Confederate Monuments ». The New York Times, 15 août 2017, nytimes.com/2017/08/15/arts/design/trump-robert-e-leegeorge-washington-thomas-jefferson.html. Page consultée le 22 septembre 2016.

SINHA, Manisha. The Slave's Cause: A History of Abolition. New Haven, CT. : Yale University Press, 2016.

SPLC (Southern Poverty Law Center). "Whose Heritage? Public Symbols of the Confederacy ", 21 avril 2016, splcenter.org/20160421/whose-heritage-public-symbols-confederacy. Page consultée le 22 septembre 2016.

STATE OF MISSISSIPPI. «A Declaration of the Immediate Causes which Induce and Justify the Secession of the State of Mississippi from the Federal Union ", 9 janvier 1861, avalon.law.yale.edu/19th_century/csa_missec.asp. Page consultée le 22 septembre 2016.

STEPHENS, Alexander H. « Cornerstone Speech », Savannah, Georgia, 21 mars 1861, teachingamericanhistory.org/library/document/cornerstone-speech/. Page consultée le 22 septembre 2016 .

TIN, Louis-Georges. « Mémoire de l'esclavage : Débaptisons les collèges et les lycées Colbert! ». Le Monde, 17 septembre 2017, lemonde.fr/idees/article/2017/09/17/debaptisons-les-colleges-et-leslycees-colbert_5186813_3232.html. Page consultée le 22 septembre 2016.

VANN WOODWARD, C. The Strange Career of Jim Crow. New York : Oxford University Press, 1955. 
VIDAL, Cécile. Éd. Français ? La nation en débat entre colonies et métropole, XVI ${ }^{e}$-XIX ${ }^{e}$ siècle. Paris : Éditions de l' EHESS, 2014.

WEIL Patrick, et Stéphane DUFOIX. Éd. L'esclavage, la colonisation, et après... France, États-Unis, Grande-Bretagne. Paris : PUF, 2005.

WILLIAMS, Thomas Chatterton. "The French Origins of the 'You Will ot Replace Us' ». The New Yorker, 4 décembre 2017, newyorker.com/magazine/2017/12/04/the-french-origins-of-you-willnot-replace-us. Page consultée le 22 septembre 2016.

WILLIAMS, Thomas Chatterton, et Marc-Olivier BEHRER. « Comment l'extrême droite française influence l"alt-right' américaine ». Le Monde, 5 décembre 2017, lemonde.fr/idees/article/ 2017/12/05/comment-l-extreme-droite-francaise-influence-l-alt-rightamericaine_5225016_3232.html. Page consultée le 22 septembre 2016.

ZINSOU, Lionel, et Marc-René BAYLE. « Rapport de préfiguration de la Fondation pour la mémoire de l'esclavage, de la traite et de leurs abolitions », 8 mars 2017, gouvernement.fr/sites/ default/files/document/document/2017/03/

rapport_de_prefiguration_de_la_fondation_pour_la_memoire_de_lesclavage_de_la_traite_et_de_leurs_abolitions.pdf. Page consultée le 22 septembre 2016.

\section{NOTES}

1. Je remercie Nicolas Barreyre et Guillaume Marche pour leurs précieuses remarques sur des versions antérieures de ce texte. Les erreurs et imprécisions qui y subsisteraient ne sauraient leur être imputées.

INDEX

Thèmes : Perspectives

\section{AUTEUR}

\section{NICOLAS MARTIN-BRETEAU}

Université de Lille 